\title{
Biopsia hepática en la patología del trasplante, período postrasplante temprano, enfoque dirigido al diagnóstico histopatológico y su correlación clínico- patológica
}

\section{Liver Biopsies in Transplant Pathology: Histopathological Diagnosis and Clinicopathological Correlation in the Early Post-Transplant Period}

Rocío del Pilar López Panqueva, MD. ${ }^{1}$

1 Patóloga, Hospital Universitario Fundación Santa fe de Bogotá, Facultad de Medicina Universidad de Los Andes.

Fecha recibido: $29-04-16$ Fecha aceptado: 03-05-16

\begin{abstract}
Resumen
La biopsia hepática de los aloinjertos sigue siendo considerada el estándar de oro y juega un papel importante e integral en la interpretación y explicación de los cambios que puedan ocurrir en respuesta a alteraciones en las pruebas de la función o bioquímica hepática, anomalías funcionales o alteración en las imágenes diagnósticas, las cuales pueden, o no, ir acompañadas de síntomas. También es útil en el seguimiento o biopsias por protocolo (1-3). La evaluación de biopsias, después del trasplante, puede ser difícil debido a que es muy amplio el espectro de las complicaciones que pueden presentarse en el período postrasplante; más aún, cuando muchas de ellas necesitan un diagnóstico y tratamiento inmediato. La patología más frecuente es el rechazo agudo. Sin embargo, también pueden observarse cambios de perfusión/reperfusión, alteraciones funcionales, recidiva de enfermedad de base, lesión de la vía biliar, lesiones vasculares, infecciones oportunistas, patologías de novo, como la hepatitis autoinmune, hepatitis crónica idiopática postrasplante, toxicidad farmacológica o tumores, entre otras patologías (4). En este artículo relacionado con la patología del trasplante hepático se tratarán las patologías más frecuentes, no quirúrgicas, en el período postrasplante temprano, con un enfoque histopatológico dirigido a las dificultades y controversias para una adecuada correlación clínico-patológica.
\end{abstract}

\section{Palabras clave}

Trasplante hepático, biopsia hepática, disfunción del injerto, isquemia de perfusión/reperfusión, rechazo del aloinjerto, rechazo agudo humoral, C4d, rechazo agudo celular, inflamación portal, ductulitis, endotelitis, endotelialitis.

\begin{abstract}
Biopsies of liver allografts are still considered to be the gold standard. They play an important and integral role in the interpretation and explanation of changes that may occur in response to alterations in function tests, in the interpretation and explanation of liver biochemistry, in the interpretation and explanation of functional abnormalities, and in the interpretation and explanation of diagnostic images (whether or not accompanied by symptoms). Biopsies are also useful for monitoring and are often part of the protocol (1-3). The evaluation of biopsy samples after transplantation can be difficult especially because of the very broad spectrum of complications that may arise in the post-transplant period. Many of them require immediate diagnosis and treatment despite this difficulty. Although the most common condition is acute rejection, many other conditions and disorders can be observed. They include perfusion/reperfusion alterations, functional impairment, recurrence of underlying diseases, injury to the bile duct, vascular lesions, opportunistic infections, de novo pathologies such as autoimmune hepatitis, posttransplant idiopathic chronic hepatitis, drug toxicity, and tumors (4). This is the second article about the pathology of liver transplantation. It discusses the most common pathologies in the early post-transplant period and provides a histopathological approach towards difficulties and controversies for adequate clinicopathological correlation.
\end{abstract}

\section{Keywords}

Liver transplantation, liver biopsy, graft dysfunction, perfusion ischemia, reperfusion injury, allograft rejection, acute humoral rejection, C4d, acute cellular rejection, portal inflammation, ductulitis, endotheliitis, endothelialitis 


\section{INTRODUCCIÓN}

Tradicionalmente la patología del postrasplante hepático se ha dividido según su tiempo de aparición sea temprana o tardía; algunas de estas se resumen en la Tabla 1. Las preguntas que nos hacemos con más frecuencia dependen del tiempo en el cual ocurre el evento al cual nos enfrentamos. Si durante la primera semana postrasplante hay empeoramiento de la función hepática, o no se normalizan las pruebas, estamos ante una disfunción primaria o secundaria del injerto y deben ser considerados: la lesión de preservación/reperfusión, problemas inmunológicos como rechazo agudo celular, incompatibilidad $\mathrm{ABO}$ o rechazo mediado por anticuerpos; factores que dependen del donante o de los problemas técnicos, en especial vasculares por falla en la anastomosis o trombosis.

Tabla 1. Complicaciones no quirúrgicas más frecuentes del hígado trasplantado

\begin{tabular}{|c|c|c|}
\hline Patología & Frecuencia & $\begin{array}{l}\text { Tiempo/pico de } \\
\text { presentación } \\
\text { postrasplante }\end{array}$ \\
\hline $\begin{array}{l}\text { Rechazo hiperagudo, } \\
\text { rechazo humoral }\end{array}$ & $\begin{array}{l}\text { Menos } 0,1 \% \text {, muy } \\
\text { raro }\end{array}$ & $\begin{array}{l}\text { Primeras } 2 \text { semanas: } \\
\text { 1-14 días }\end{array}$ \\
\hline Rechazo agudo celular & $15 \%-80 \%$ & Primer año: 5-30 días \\
\hline $\begin{array}{l}\text { Rechazo crónico } \\
\text { (ductopénico y } \\
\text { vascular) }\end{array}$ & Menos 3\% & $60-180$ días \\
\hline $\begin{array}{l}\text { Colangiopatía } \\
\text { isquémica }\end{array}$ & $10 \%-19 \%$ & 30-180 días \\
\hline $\begin{array}{l}\text { Infecciones } \\
\text { oportunistas }\end{array}$ & $\begin{array}{l}\text { Variable, según } \\
\text { microorganismo } \\
\text { entre } 1 \%-42 \%\end{array}$ & 3-6 meses \\
\hline $\begin{array}{l}\text { Toxicidad } \\
\text { medicamentosa }\end{array}$ & No bien establecida & $\begin{array}{l}\text { Cualquier momento } \\
\text { postrasplante }\end{array}$ \\
\hline $\begin{array}{l}\text { PTLD, malignidad de } \\
\text { novo }\end{array}$ & $\begin{array}{l}0,9 \%-2,9 \%(4 \%) \\
3 \%-5 \% \\
11-20 \%\end{array}$ & $\begin{array}{l}\text { Primer año } \\
\text { Primeros } 3 \text { años } \\
10 \text { años }\end{array}$ \\
\hline \multicolumn{3}{|c|}{ Recurrencia de patología de base } \\
\hline HVC & Más 90\% & 60-120 días \\
\hline HVB & Menos $10 \%$ & 30-180 días \\
\hline PBC & $18 \%(4 \%-33 \%)$ & 1-10 años \\
\hline PSC & $50 \%$ & 1-5 años \\
\hline $\mathrm{NASH}$ & $40 \%$ & \\
\hline Alcohol & $10 \%-90 \%$ & Primeros 5 años \\
\hline $\mathrm{HCC}$ & $10 \%$ & 1-5 años \\
\hline $\begin{array}{l}\text { Complicaciones } \\
\text { metabólicas: } \\
\text { hipertensión, } \\
\text { diabetes mellitus } \\
\text { (DM), hiperlipidemia, } \\
\text { obesidad y gota }\end{array}$ & $5 \%-26 \%$ & $\begin{array}{l}\text { Inicio desde el primer } \\
\text { año }\end{array}$ \\
\hline
\end{tabular}

\section{LESIONES TEMPRANAS OBSERVADAS EN EL HÍGADO TRASPLANTADO}

\section{Disfunción del injerto}

No existe una definición clara de este término; sin embargo, su característica principal es una deficiente función del injerto en las primeras semanas del postrasplante, lo cual augura una pobre sobrevida del injerto y por ende del paciente. Se refleja clínicamente por la presencia de una elevación importante de las aminotransferasas y una prolongación de los tiempos de coagulación. Cuando no existe riesgo vital, corresponde a una pobre función del injerto; en esta disfunción precoz hay una elevación muy severa de las transaminasas, usualmente mayor a $2000 \mathrm{U} / \mathrm{L}$, acompañado de hiperbiblirrubinemia mayor de $10 \mathrm{mg} / \mathrm{dL}$.

$\mathrm{Si}$ el riesgo es vital, se encuentran valores de transaminasas por encima de $5000 \mathrm{U} / \mathrm{L}$, con prolongación del índice internacional normalizado (INR) tres o más veces su valor normal y acidosis metabólica. En esta situación se habla de fallo primario del injerto y es considerada una emergencia que requiere retransplante. De lo contrario, la probabilidad de muerte es muy alta $(5,6)$. Algunos estudios han mostrado asociación en el desarrollo de la disfunción temprana con el estado preoperatorio del receptor, en especial si este ha requerido uso de ventilación asistida, la donación tras la muerte cardíaca, la edad del donante, el tamaño del injerto, el grado de esteatosis, el tiempo operatorio y la necesidad de transfusión intraoperatoria $(7,8)$.

\section{Isquemia de perfusión/reperfusión}

El proceso de preservación del órgano y su ulterior reperfusión conlleva invariablemente a una lesión celular. El principal factor etiológico de la disfunción primaria del injerto (DPI) se relaciona con la lesión de isquemia de reperfusión, la cual se produce inmediatamente el oxígeno vuelve a circular en forma rápida en un órgano isquémico; esta isquemia produce disminución de nutrientes y energía, lo que altera la homeostasis, se inicia a través de la generación especies de oxígeno reactivas (ROS) por los hepatocitos durante la isquemia activando vías de señalización implicadas en la respuesta hipóxica y al mismo tiempo una respuesta inmune de tipo inflamatorio que activa las células de Kupffer, linfocitos CD4+ y polimorfonucleares neutrófilos permitiendo la migración hacia el sitio de la lesión y la activación de citocinas, quimiocinas y proteínas del complemento, donde previamente, por la generación de grandes cantidades de ROS, lleva a apoptosis celular y muerte celular con necrosis de tipo isquémico luego de la reperfusión. Además ocurren lesiones endoteliales que dañan la microcirculación y favorecen el desarrollo de fenó- 
menos trombóticos, con la consiguiente necrosis del tejido hepático (9-11). Es una causa importante de la disfunción del injerto en el postoperatorio temprano, hasta $50 \%$ de los aloinjertos muestran algún signo de daño isquémico, y representa el $10 \%$ de falla temprana del órgano; es mayor la probabilidad de presentarse si existen lesiones preestablecidas en el órgano donante, por ejemplo esteatosis macrovesicular o siderosis (12).

Histológicamente, se caracteriza por edema celular o balonización, necrosis aislada con formación de cuerpos acidofílicos o necrosis masiva acompañada de agregados de neutrófilos y colestasis hepatocanalicular de localización perivenular (zona 3), dada la mayor susceptibilidad de esta región a los eventos isquémicos, los cambios se hacen evidentes en las primeras 48 horas después del trasplante (Figuras 1 y 2). Cuando se toman biopsias en tiempo cero (0), o posreperfusión, son más prominentes los agregados de células inflamatorias polimorfonucleares neutrófilos. En casos severos, disminuye la inflamación y aumenta la necrosis, lo que compromete todo el ácino (panacinar) (13).

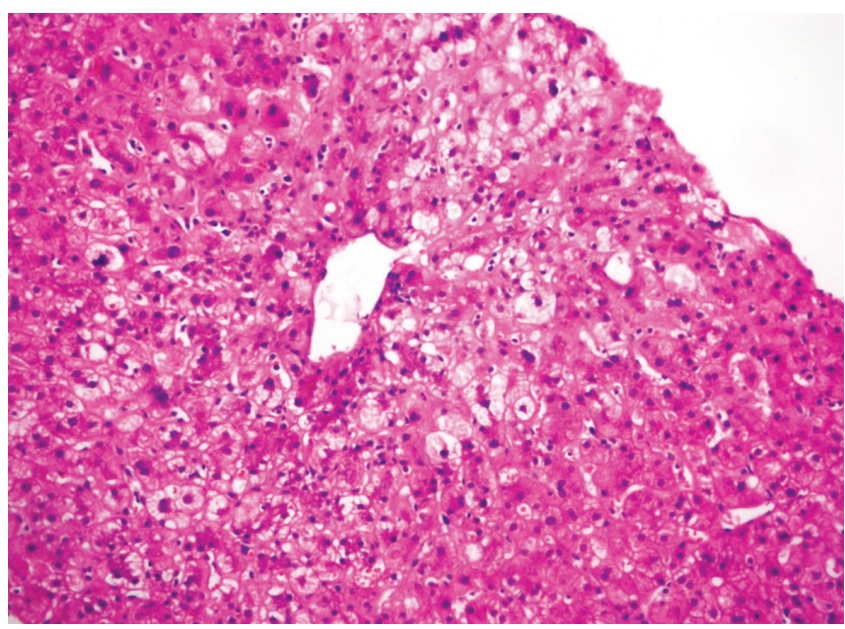

Figura 1. Hematoxilina \& Eosina 20x. Lesión de perfusión/reperfusión. Nótese el marcado edema celular perivenular.

Menos frecuentemente pueden observarse necrosis en región periportal o infartos subcapsulares. Cuando hay donante con hígado graso, la lesión de reperfusión favorece la ruptura de hepatocitos, lo que libera glóbulos de grasa, que se quedan atrapados en los cordones de hepatocitos y causan obstrucción, semejando sinusoides dilatados o lipopeliosis, los cuales pueden migrar al pulmón causando embolia grasa. El pronóstico de esta condición depende directamente del porcentaje de parénquima con necrosis (14). Se incluyen en el diagnóstico diferencial el rechazo humoral, rechazo celular y complicaciones quirúrgicas, en especial de anastomosis vascular o de la vía biliar. La colestasis de presentación muy temprana también puede deberse a injertos pequeños para el donante (small-forsize), casos en los que el edema celular es muy prominente y de localización no solo perivenular sino que puede ser difusa o panacinar.

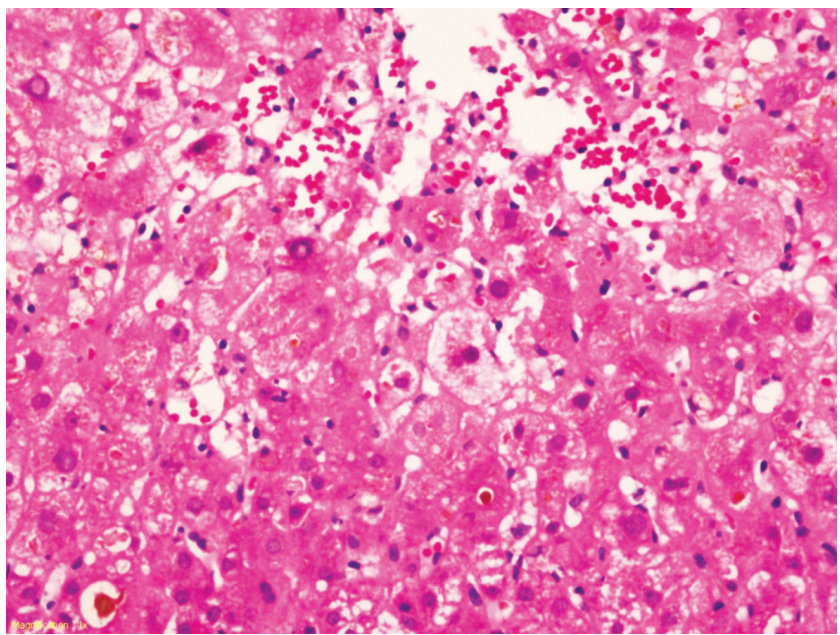

Figura 2. Hematoxilina \& Eosina 40x. Lesión de perfusión/reperfusión. Hay marcada balonización, colestasis hepatocanalicular de localización perivenular.

\section{RECHAZO DEL ALOINJERTO}

Desde 1995 con la introducción de la clasificación de Banff por parte del consenso internacional y después, en 1997, el panel de expertos, con el ánimo de definir y estandarizar criterios para el diagnóstico histopatológico del rechazo en trasplante, se caracterizaron y clasificaron las lesiones relacionadas. Hasta la fecha, han sido aceptadas: el rechazo humoral, rechazo agudo celular y rechazo crónico $(15,16)$. En este artículo nos centraremos en las dos primeras.

\section{Rechazo humoral}

El rechazo agudo humoral es muy raro entre los trasplantes hepáticos $\mathrm{ABO}$ compatibles. Inicialmente fue considerado incompatible con el trasplante hepático, sin embargo ya es reconocido como causa muy probable de lesión, tanto temprana como tardía. Su verdadera incidencia ha sido difícil de establecer por la falta de estudios que permitan correlacionar la presencia de $\mathrm{C} 4 \mathrm{~d}$ con los anticuerpos antidonante específicos (17). El rechazo primario mediado por anticuerpos y/o complemento que ocurre inmediatamente (pocas horas o días) es el llamado rechazo hiperagudo; si ocurre unos pocos días después o durante la primera semana se habla de rechazo humoral agudo o rechazo agudo mediado por anticuerpos postrasplante. La forma secundaria ocurre cuando se desarrollan anticuerpos de novo. Los anticuerpos pueden ser preformados o corresponder a anticuerpos anti- 
donante específicos desarrollados después de un trasplante. Los anticuerpos reaccionan con los antígenos del donante y pueden tener efectos diferentes en el aloinjerto, desde producir su destrucción, favorecer la sobrevida o no tener ninguna alteración en su función sintética. Tienen influencia en este desenlace la clase, el título y la especificidad de los anticuerpos antidonante específico; también la densidad y distribución de los antígenos diana en el órgano receptor. Se han descrito especialmente los anticuerpos dirigidos contra los antígenos principales de los grupos sanguíneos $\mathrm{ABO}$ y antígenos $\mathrm{MHC}$, cualquiera de ellos pueden causar rechazo del aloinjerto $(18,19)$. Clínicamente se caracteriza por una elevación progresiva de la bilirrubina y trombocitopenia marcada, asociada con signos de insuficiencia hepática.

En el examen anatomopatológico, en las fases más iniciales, los cambios son inespecíficos, semejan lo observado en la lesión de preservación con balonización de hepatocitos y necrosis hepatocitaria en zona 3 . El endotelio es la célula directamente lesionada; por lo tanto, el daño microvascular que se produce en las primeras horas lleva a hemorragia focal con agregados sinusoidales de células inflamatorias de tipo polimorfonucleares neutrófilos y plaquetas, con trombosis de vasos pequeños. Progresa a edema portal y periportal con reacción ductular o proliferación colangiolar, lo que semeja obstrucción de la vía biliar, con hipertrofia de células endoteliales que compromete vasos venosos portales y capilares, acompañado de linfocitos y eosinófilos adheridos al endotelio (Figura 3).

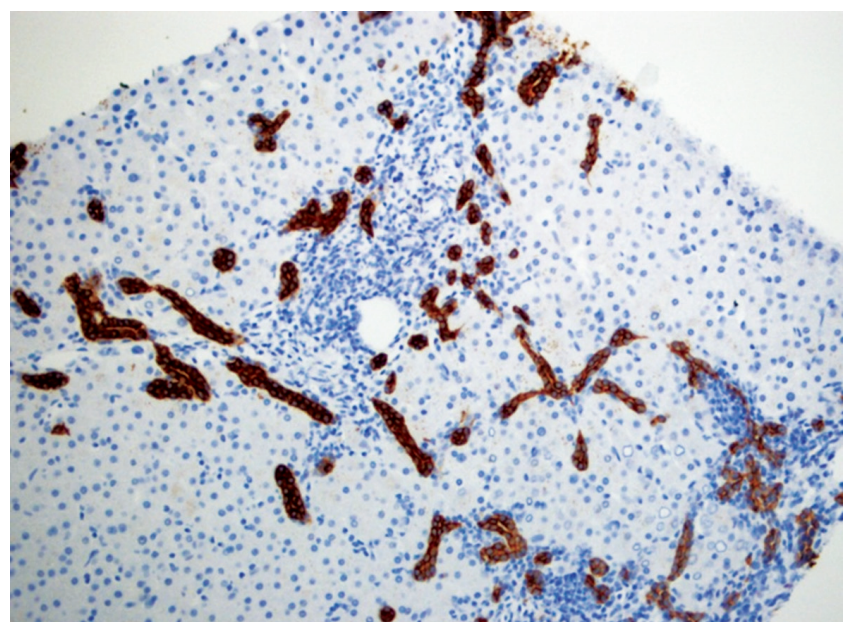

Figura 3. Estudios de Inmunohistoquímica para citoqueratina 7 realza la marcada proliferación de colangiolos, rodeando los espacios portales inflamados.

En casos severos puede existir trombosis de las ramas intrahepáticas de la vena portal, con infartos venosos y cambios extensos de tipo isquémico y formación de infartos geográficos; puede haber compromiso de venas y arterias hepáticas o de la vena cava inferior. Para este diagnóstico es indispensable asegurarse de excluir trombosis de la arteria hepática o la obstrucción biliar. Reactividad para $\mathrm{C} 4 \mathrm{~d}$ por inmunohistoquímica (en tejido incluido en parafina) es difícil de interpretar aisladamente; tiene varios patrones, debe ser intensa en al menos el 50\% de las venas portales, en capilares del estroma portal o con distribución sinusoidal (Figura 4). Si existe disponibilidad de tejido en fresco o congelado, el método considerado más confiable son estudios de inmunofluorescencia con reactividad sinusoidal para C4d. También se ha demostrado reactividad linear para IGG o IGM, fracciones del complemento C3, C4, C1q, fibrinógeno en la pared de los vasos arteriales y presencia de anticuerpos anti HLA $(17,20-24)$. Es importante enfatizar que se ha descrito presencia focal de C4d en una gran cantidad de aloinjertos, sin evidencia de rechazo humoral mediado por anticuerpos. Por lo tanto, el diagnóstico preciso de rechazo humoral puede ser útil para determinar e indicar la posibilidad del uso de plasmaféresis en el postoperatorio inmediato o mediato, y para evaluar en los protocolos actuales la posibilidad de trasplante $\mathrm{ABO}$ incompatible (24).

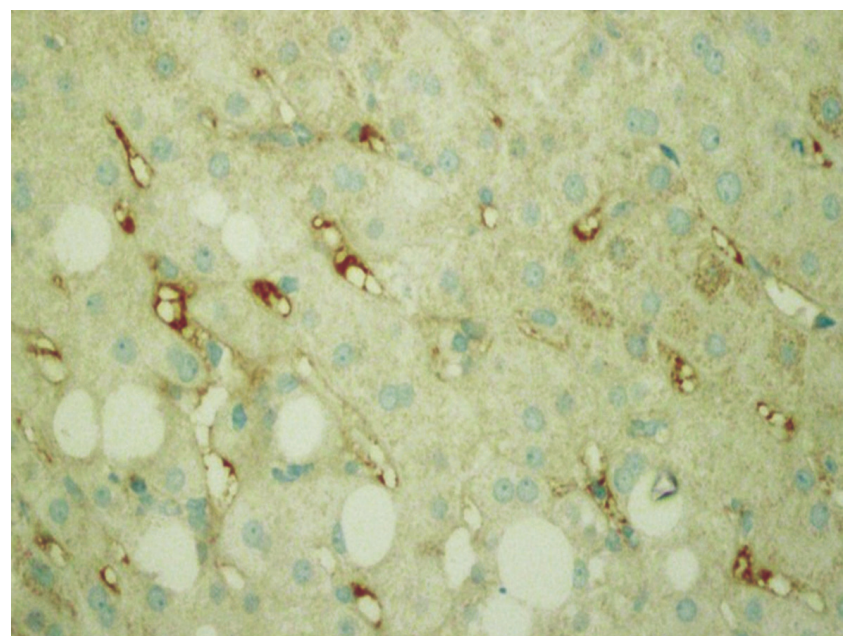

Figura 4. Estudio de Inmunohistoquímica para C4d con reactividad (coloración café) revistiendo sinusoides.

\section{Claves para el diagnóstico de rechazo humoral}

- Disfunción del injerto por clínica y laboratorios

- Biopsia hepática con evidencia de daño activo del injerto que sugiera rechazo humoral

- Reactividad intensa para C4d en cualquier patrón

- Presencia de anticuerpos anti-donante específicos circulantes 


\section{Rechazo agudo celular}

El rechazo agudo celular es una respuesta inflamatoria linfocitaria inmunomediada, dependiente de las células $\mathrm{T}$. $\mathrm{Su}$ riesgo ha sido contrarrestado por el uso adecuado de la inmunosupresión. Hoy en día, la mortalidad y morbilidad debida al rechazo del injerto es un evento poco común, pero sigue siendo la principal causa de la disfunción del injerto; Se presenta entre el 50\%-75\% de todos los pacientes trasplantados. Puede ocurrir desde las primeras semanas del postrasplante, la mayoría de ellos $(60 \%)$ ocurre en los primeros 6 meses después del trasplante, en especial durante los primeros 5-30 días. Sin embargo, un rechazo agudo puede presentarse en cualquier momento del postrasplante, incluso varios años después se presenta en pacientes con una mala adherencia al tratamiento inmunosupresor, por interacción con otros medicamentos o con otros factores que alteren los niveles séricos; por ejemplo, de los inhibidores calcineurínicos, en receptores mujeres y con enfermedades autoinmunes. La biopsia juega un papel fundamental en el diagnóstico y manejo del mismo $(3,4,25)$.

La calificación del sistema Banff asigna un peso numérico a cada uno de los criterios o parámetros morfológicos, según su severidad, valorando un índice de actividad bien conocido como RAI score para el rechazo agudo celular, asigna una puntuación de 1 a 3 con máximo posible de 9 . En la Tabla 2 se indica una aproximación a la evaluación patológica utilizando un índice semicuantitativo; en la Tabla 3, la graduación y terminología utilizada según recomendación del consenso de Banff. La aplicación del esquema de Banff presupone que el diagnóstico histológico ya se ha establecido $(16,26)$. La triada clásica incluye inflamación portal, lesión de ductos biliares portales y lesión inflamatoria vascular. Es necesario tener al menos dos de estas tres características; la presencia de inflamación portal es indispensable para el diagnóstico histopatológico de rechazo agudo (16). Los criterios histopatológicos que debemos tener en cuenta se evalúan en densidad de la inflamación y en porcentaje de compromiso de los espacios portales representados en la biopsia, o de las estructuras ductales o vasculares comprometidas. Algunos autores recomiendan como mínimo una representación de 7 espacios porta.

- Inflamación portal: cualquier grado de inflamación portal con presencia de una población heterogénea de células inflamatorias, tales como linfocitos $\mathrm{T}$ activados, inmunoblastos y eosinófilos, acompañados de algunos plasmocitos y polimorfonucleares neutrófilos. Puede existir leve o mínima inflamación lobulillar, en parches (Figura 5)

- Lesión inflamatoria de la vía biliar: ductulitis o presencia de células inflamatorias linfoides en el epitelio de ductos pequeños $(<30 \mu \mathrm{m})$ y cambios degenerativos dados por aumento de la relación núcleo/citoplasma, hipercromasia y pleomorfismo nuclear, seudoestratificación nuclear o alteración de la polaridad, mitosis, vacuolización paranuclear del epitelio ductal; puede existir ruptura de la membrana basal (Figura 6).

- Inflamación vascular endotelial: no está presente en todos los casos, se localiza a nivel portal y/o en la vena hepática terminal o central, con inflamación subendotelial de linfocitos, prominencia y desprendimiento endotelial. Endotelialitis sinusoidal muy ocasionalmente puede estar presente (Figura 7).

Tabla 2. Puntuación del rechazo agudo celular, RAI score

\begin{tabular}{|c|c|c|}
\hline Categorías principales & Criterios morfológicos & Calificación \\
\hline \multirow[t]{3}{*}{ Inflamación portal } & Inflamación de linfocitos que compromete una minoría de las triadas. & 1 \\
\hline & $\begin{array}{l}\text { Expansión de la triada portal por inflamación de linfocitos, plasmocitos, neutrófilos, eosinófilos y } \\
\text { algunos blastos. }\end{array}$ & 2 \\
\hline & $\begin{array}{l}\text { Marcada expansión portal por infiltrado inflamatorio heterogéneo con numerosos blastos y } \\
\text { eosinófilos en todas o en la gran mayoría de las triadas portales y extensión a la zona periportal. }\end{array}$ & 3 \\
\hline \multirow{3}{*}{$\begin{array}{l}\text { Inflamación y lesión del } \\
\text { ducto biliar }\end{array}$} & Ocasionales ductos muestran inflamación intraepitelial y leves cambios de tipo reactivo. & 1 \\
\hline & La mayoría de los ductos muestran inflamación y cambios ductales de tipo degenerativo. & 2 \\
\hline & $\begin{array}{l}\text { Compromiso de todos los ductos por células inflamatorias y cambios degenerativos severos del } \\
\text { epitelio ductal, con disrupción de la luz. }\end{array}$ & 3 \\
\hline \multirow{3}{*}{$\begin{array}{l}\text { Inflamación venosa } \\
\text { endotelial }\end{array}$} & Infiltración subendotelial de linfocitos que compromete algunas vénulas portales y/o hepáticas. & 1 \\
\hline & $\begin{array}{l}\text { Inflamación subendotelial de linfocitos en la mayoría de las vénulas hepáticas y/o portales, } \\
\text { desprendimiento de células endoteliales. }\end{array}$ & 2 \\
\hline & $\begin{array}{l}\text { Inflamación subendotelial de linfocitos moderada o severa de venas hepáticas y/o portales, } \\
\text { compromiso de venas centrales, desprendimiento endotelial y necrosis perivenular. }\end{array}$ & 3 \\
\hline
\end{tabular}


Tabla 3. Escala de Banff para el rechazo agudo celular

\begin{tabular}{ll}
\hline Evaluación global & Criterios \\
\hline $\begin{array}{l}\text { No rechazo, puntaje 0-1 } \\
\text { Indeterminado 0 } \\
\text { borderline: puntaje 2-3 } \\
\text { Leve: puntaje 3-4 } \\
\text { (grado I) }\end{array}$ & $\begin{array}{l}\text { Infiltrado cumple criterios portal de linfocitos mínima que diagnóstico. } \\
\text { celular (heterogéneo) en la minoría de las } \\
\text { triadas portales, confinado al espacio portal. }\end{array}$ \\
Moderado: puntaje 5-7 & $\begin{array}{l}\text { Infiltrado característico de rechazo agudo } \\
\text { celular en la mayoría de las triadas portales. }\end{array}$ \\
$\begin{array}{l}\text { Severado II) } \\
\text { (grado III) }\end{array}$ & $\begin{array}{l}\text { Infiltrado característico de rechazo agudo } \\
\text { celular en casi todas las triadas portales, } \\
\text { de densidad celular moderada a severa, } \\
\text { que se extiende a la región periportal, se } \\
\text { extiende al parénquima hepático y con } \\
\text { necrosis perivenular. }\end{array}$ \\
\hline
\end{tabular}

En rechazo severo puede existir arteritis necrotizante, aunque es infrecuente en las biopsias, ya que usualmente compromete vasos hiliares.

\section{Otros patrones de rechazo agudo celular}

\section{Perivenulitis central o venulitis central}

Se observa hasta en el $30 \%$ de los casos, también es más frecuente en el rechazo agudo tardío (después del día 100) y en rechazos pediátricos. Se caracteriza por la endotelitis o inflamación de las vénulas terminales; a veces acompañado de congestión, extravasación de hematíes y dilatación sinusoidal, con leve o mínima inflamación, hepatocitos apoptóticos aislados en su vecindad (zona 3) (Figura 8). A diferencia del rechazo agudo celular clásico, el endotelio usualmente no presenta cambios de endotelialitis; en la mayoría de los casos aparece esencialmente normal. Puede acompañarse de rechazo agudo celular con cambios infla- matorios a nivel portal o presentarse como venulitis central aislada $(27,28)$.

\section{Rechazo basado en inflamación lobulillar o patrón Iobulillar}

En ocasiones la inflamación del lobulillo o hepatitis lobulillar domina el cuadro morfológico, con mínimos cambios a nivel portal o presencia de cambios inespecíficos. Se observa especialmente en niños; también se ha descrito en los adultos que suspenden abruptamente inmunosupresión después de estar recibiéndola plenamente. El lobulillo muestra infiltrado de linfocitos (hepatitis linfocítica) leve o en parches, con algunos cuerpos apoptóticos dispersos y células de Kupffer hiperplásicas, mínima inflamación portal, con leve ductulitis, sin evidencia de inflamación endotelial, ni tampoco compromiso de venas centrales (Figura 9). El principal diagnóstico diferencial es la recurrencia temprana de hepatitis $C$, infección por virus de EpsteinBarr (VEB) y toxicidad medicamentosa (26).

\section{Patrón infiltrativo (sinusoidal) y patrón hepatítico}

Estos patrones de rechazo celular se han observado durante el primer año postrasplante. El patrón infiltrativo muestra infiltración sinusoidal prominente de linfocitos activados CD3 (+) acompañando a la clásica triada del rechazo. En el patrón hepatítico se sobreponen las características típicas del rechazo agudo celular, acompañados de necroinflamación del parénquima o focos de necrosis confluente, sin una preferencia en su localización (Figura 10). Clínicamente, muestra más compromiso sistémico, elevación de AST y buena respuesta al tratamiento, pero tienen un potencial mayor hacia la cronicidad. No están incluidos en la puntuación de Banff, por lo que se recomienda realizar el puntaje RAI, indicando la presencia de estos patrones, lo cual requerirá un seguimiento más estrecho para evitar la
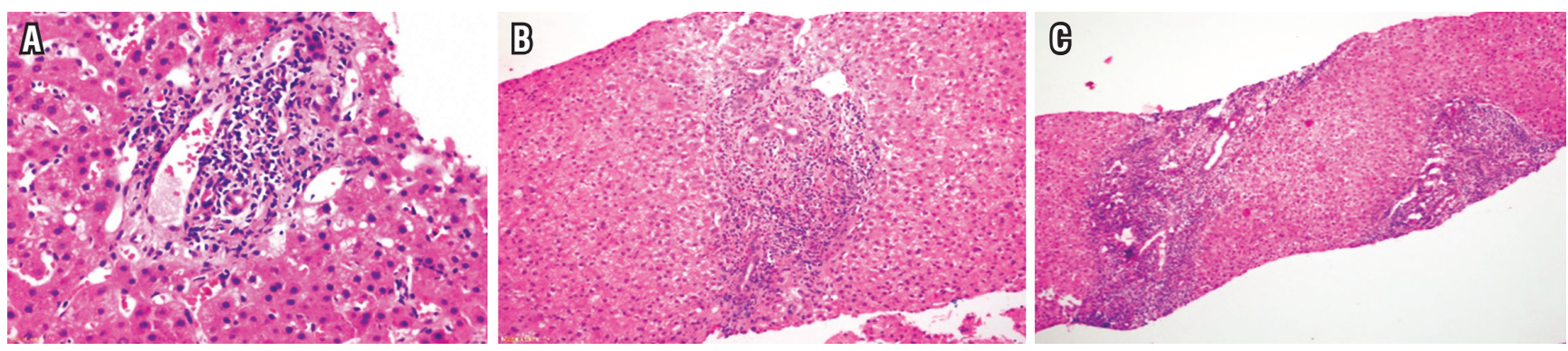

Figura 5. Hematoxilina \& Eosina, rechazo agudo celular. Inflamación portal con infiltrado inflamatorio característicamente heterogéneo, compuesto por linfocitos activados, plasmocitos, neutrófilos y eosinófilos. Intensidad A: leve (40x); B: moderada (20x); C: severa (20x). 

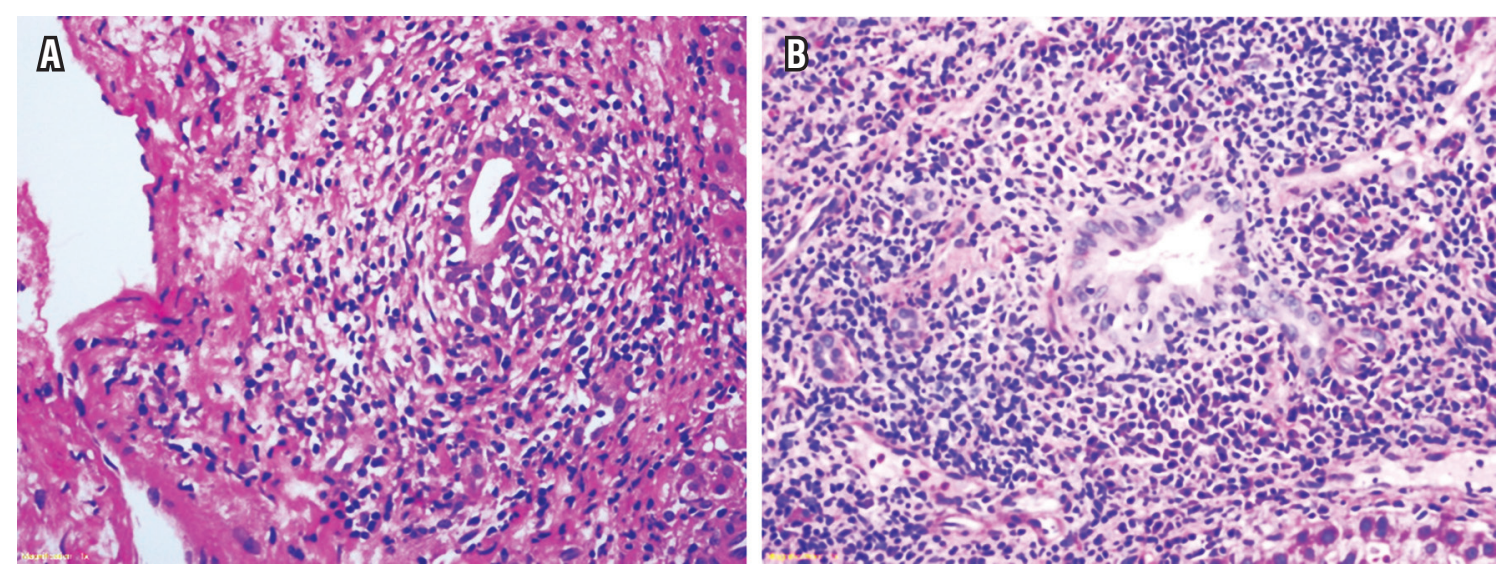

Figura 6. Hematoxilina \& Eosina 20x. Rechazo agudo celular con lesión ductal e inflamación portal. En A se observa la vacuolización del epitelio ductal con permeación de linfocitos. En B, irregularidad del contorno ductal, estratificación nuclear, hipercromasia e inflamación intraepitelial.
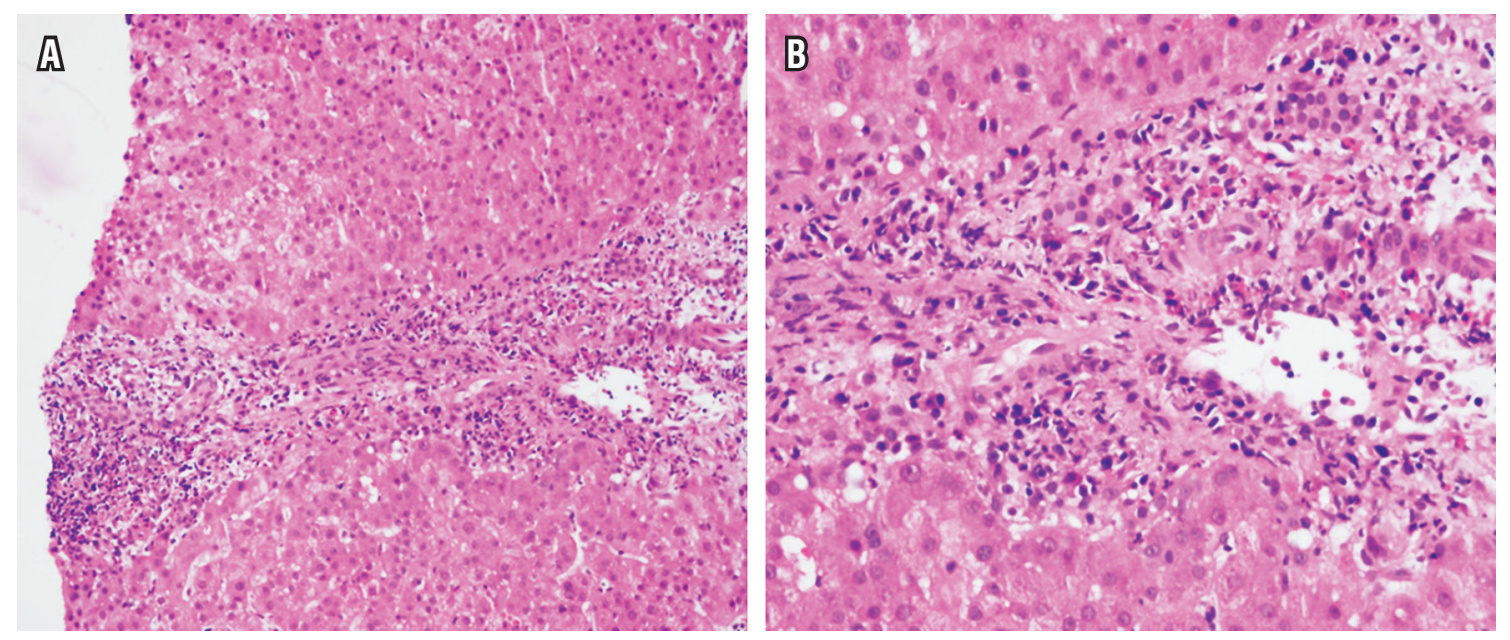

Figura 7. A. Hematoxilina \& Eosina 20x. Rechazo agudo celular. El infiltrado inflamatorio con endotelialitis, la inflamación rodea el vaso venoso portal. B. Hematoxilina \& Eosina 40x. Corresponde a un acercamiento donde se observa la prominencia de las células endoteliales y desprendimiento celular.
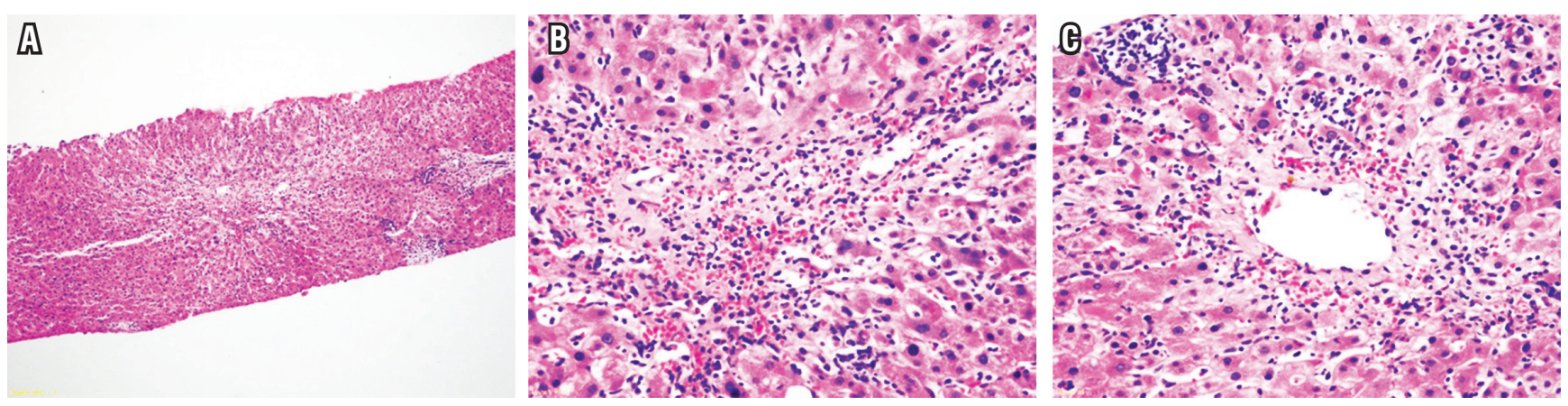

Figura 8. A. Hematoxilina \& Eosina 10x, inflamación pericentral con mínima inflamación portal. B. Hematoxilina \& Eosina 20x. C. Hematoxilina \& Eosina 40x, inflamación perivenular central. 

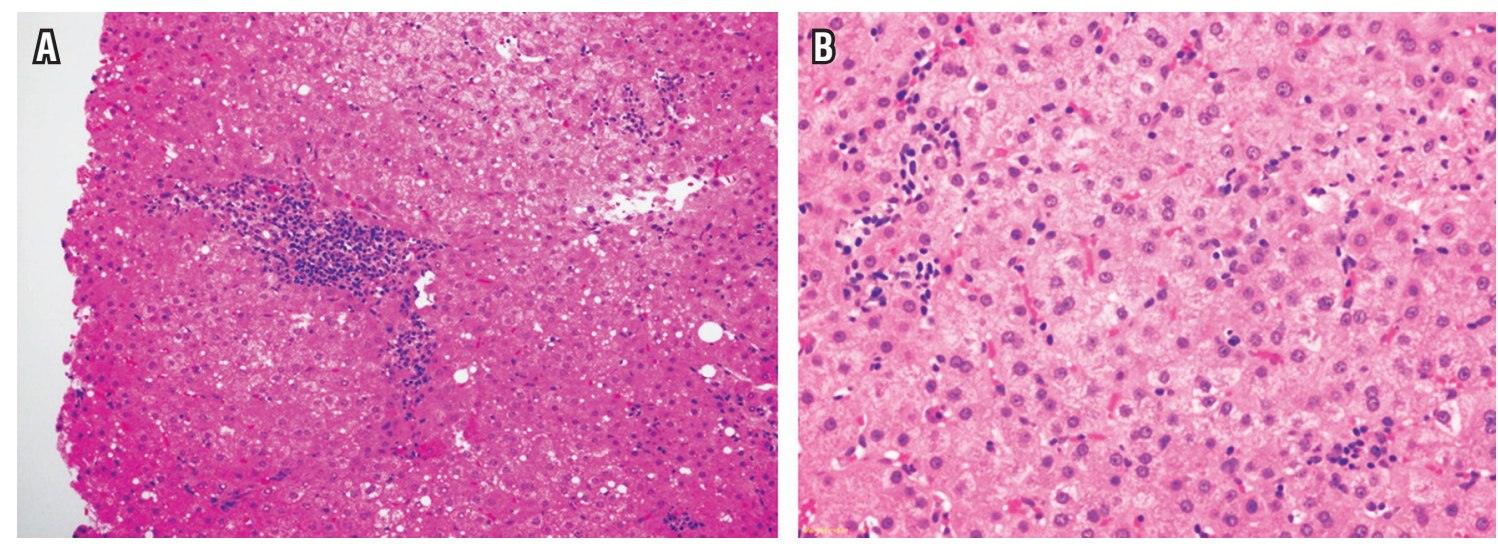

Figura 9. Hematoxilina \& Eosina. A. 10x. Además de la inflamación portal se identifican múltiples focos de inflamación lobulillar. B. 40x, infiltrado predominantemente linfocitario.
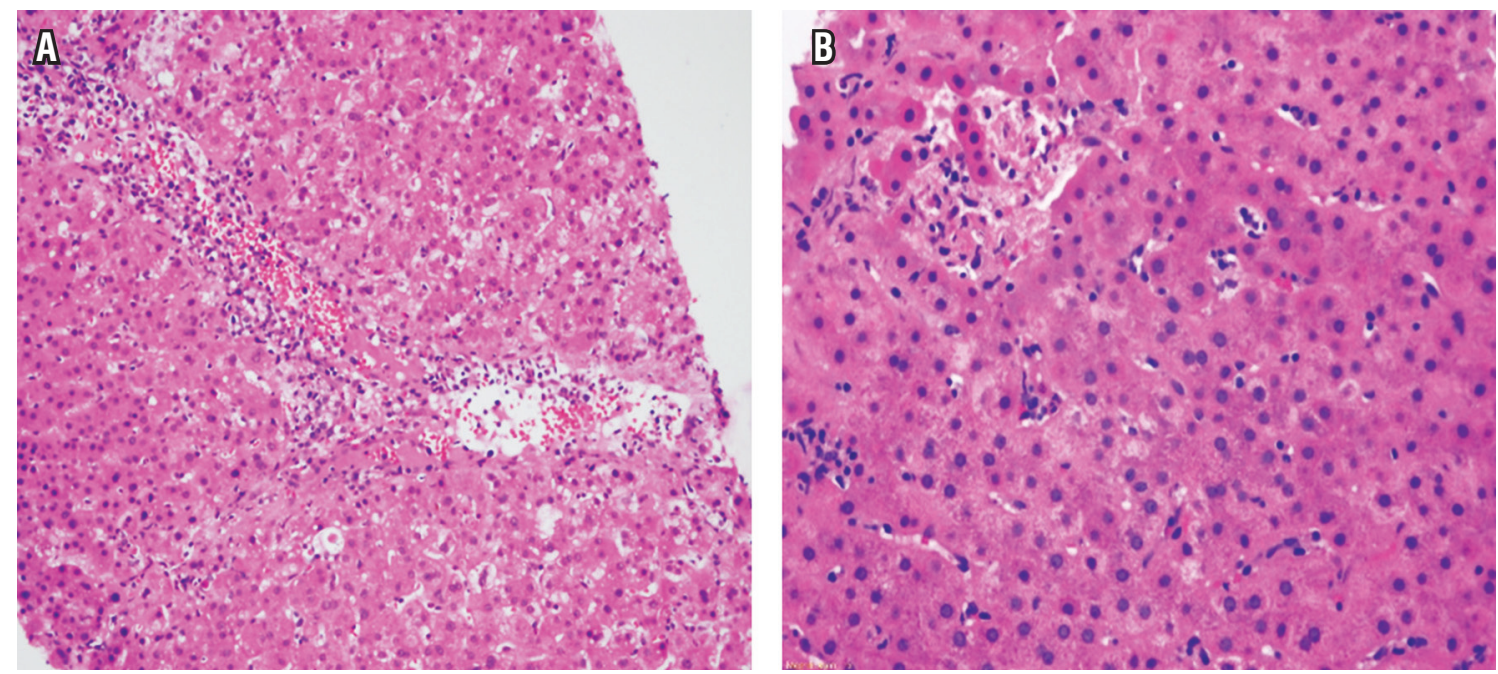

Figura 10. Hematoxilina \& Eosina 20x. A. Se observan cambios portales de rechazo celular clásico con inflamación portal, ductulitis y endotelitis asociado a inflamación lobulillar con necrosis. B. Hematoxilina \& Eosina 40x, focos dispersos de inflamación portal y zona de necrosis confuente.

progresión a rechazo crónico. El diagnóstico diferencial más importante es la presencia de infecciones virales (29).

\section{Patrón de hepatitis crónica o hepatitis idiopática postrasplante}

Característicamente, la inflamación portal es leve a moderada, con ausencia o mínima lesión ductal, con actividad de interfase presente (Figura 11). Este patrón únicamente puede ser reconocido en aquellos pacientes trasplantados que no tengan una enfermedad necroinflamatoria de base o una potencial recurrencia con este tipo de patrón, como por ejemplo la hepatitis C. Se incluyen en el diagnóstico diferencial la reacción a drogas y las otras causas de hepatitis no específica. En rechazo severo puede existir compromiso de la placa limitante con extensión del infiltrado al lobulillo e imagen de apolillamiento de la interfase. Esta última imagen se ve más en rechazo agudo tardío (más de 100 días) y es muy difícil de diferenciar de una hepatitis crónica. Este patrón se ha visto después del sexto mes postrasplante y está asociado con progresión a fibrosis $(26,30)$.

\section{Patrón con predominio de células plasmáticas}

Se observa en el contexto de un cuadro clínico y morfológico que plantea rechazo celular agudo, pero con un infiltrado predominantemente de plasmocitos (Figura 12). Hay datos limitados sobre su verdadero significado en el postrasplante hepático, pero se ha visto más asociado a una mayor gravedad histológica cuando el porcentaje de células plasmáticas corresponde a más del $30 \%$ de la población de células inflamatorias, por lo que se ha planteado la posibili- 
dad de que pueda ser utilizado como un potencial marcador de severidad. El principal problema diagnóstico es diferenciarlo de una hepatitis autoinmune (HAI) recurrente o de novo. Sin embargo, la ductulitis y endotelitis son características claves del rechazo agudo celular clásico, hallazgos que están ausentes o son mínimos en la HAI; además, de la ausencia de hepatitis de interfase, también es importante tener en cuenta que la HAI recurrente suele presentarse después de los 2 años postrasplante, mientras el rechazo variante de células plasmáticas ocurre durante los primeros 2 años. En estos pacientes se ha descrito en pocas ocasiones presencia de autoanticuerpos en títulos bajos. Se debe tener en cuenta que pacientes en tratamiento con interferón para hepatitis $C$ pueden presentar esta variante de rechazo agudo celular y muchos casos están asociados a episodios previos de rechazo agudo celular típico o en pacientes con inmunosupresión en niveles subterapéuticos (31).

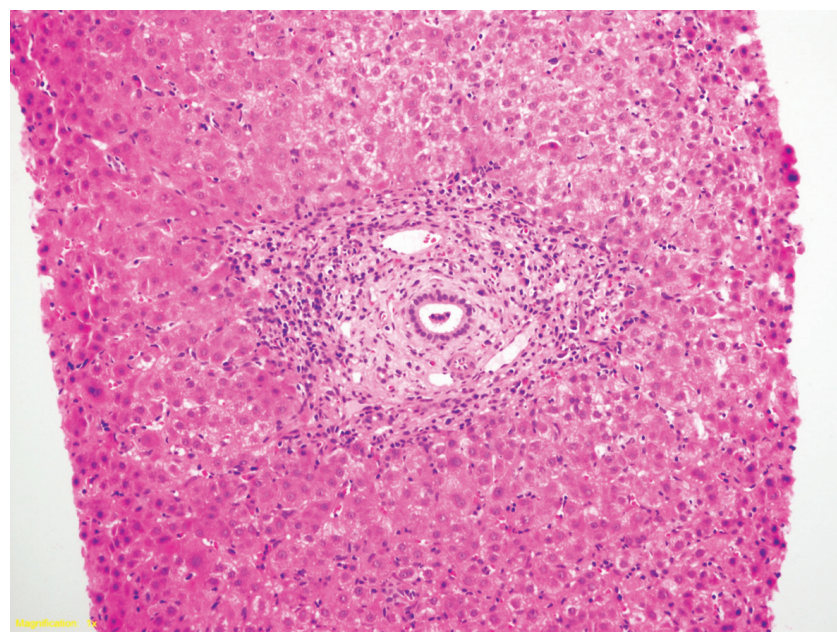

Figura 11. Hematoxilina \& Eosina 20x. Inflamación portal leve con hepatitis de interfase, nótese la irregularidad de la placa limitante con extension periportal del infiltrado.

La evidencia bioquímica de daño hepático se manifiesta como un aumento en las pruebas de función hepática, generalmente con elevación de las aminotransferasas y de la actividad de la fosfatasa alcalina o de la $\gamma$-glutamiltransferasa. En algunos casos se acompaña de leucocitosis y eosinofilia periférica. Los disparadores clínicos incluyen cualquier cambio en la inmunosupresión y condiciones que alteren el sistema inmune, por ejemplo un proceso infeccioso. Otros factores de riesgo que deben ser considerados son donantes de edad avanzada en un receptor muy joven, o condiciones preexistentes que alteren su inmunidad. Se realiza biopsia ante la sospecha clínica de un rechazo agudo o por la presencia de disfunción del injerto sin una explicación evidente, menos frecuentemente encontramos rechazo en una biopsia por protocolo $(14,26)$.

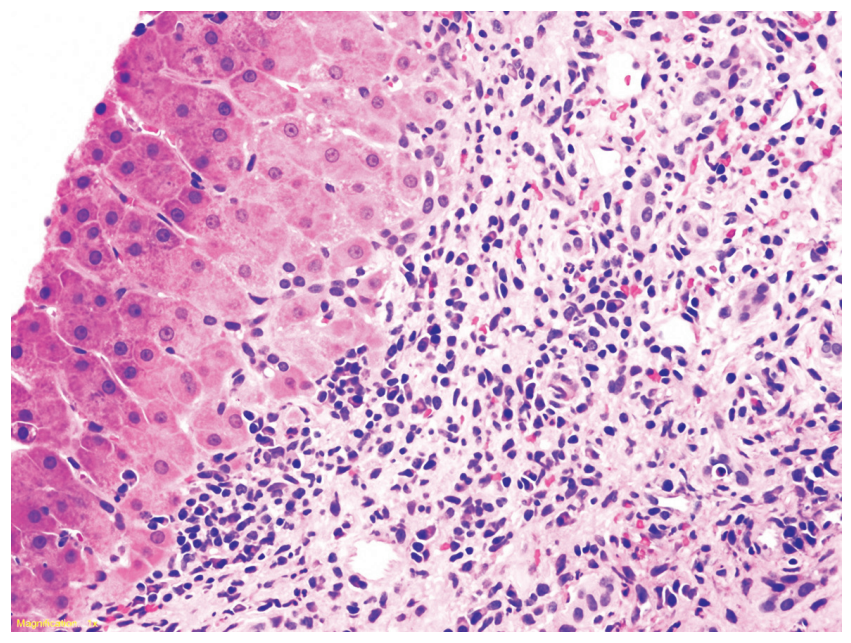

Figura 12. Hematoxilina \& Eosina 40x. Rechazo agudo celular con infiltrado inflamatorio predominantemente plasmocitario.

Es importante anotar que si la biopsia se realiza después de instaurar un tratamiento inmunosupresor, la clasificación y el índice pueden ser difíciles de establecer, en especial por la rápida modificación de la inflamación. El rechazo agudo leve en el esquema de Banff es el grado más común, corresponde a cerca del $60 \%-80 \%$ del número total de episodios y la gran mayoría de ellos responden al tratamiento con el aumento de la inmunosupresión. El rechazo agudo moderado con puntajes entre 5 y 7 ocurre en el 10\%-20\% de todos los episodios de rechazo agudo celular y son más comunes en los pacientes que tienen factores de riesgo y muestran signos o síntomas clínicos como leucocitosis o fiebre, así como alteración de las pruebas de función hepática. En menos del 5\% de los casos ocurre el rechazo severo, con mayor frecuencia se manifiesta en pacientes que no están adecuadamente inmunodeprimidos, pacientes con una prueba cruzada con títulos altos de linfocitotóxicos, antecedente de enfermedades autoinmunes o en retrasplantes. Son pacientes que invariablemente presentan síntomas y signos que pueden simular un proceso sistémico infeccioso (29,32).

Muchos de los estudios que han evaluado el valor clínico y la clasificación de Banff son contradictorios. A pesar de no ser validada totalmente, y de que no ha demostrado la posibilidad de predecir con toda certeza la sobrevida del injerto e inclusive la respuesta a esteroides, sí es ampliamente aceptada. Es considerada como un marcador útil de la gravedad del rechazo, pero es importante tener en cuenta que ni la puntuación total, ni ninguno de los criterios histopatológicos en forma individual se corresponden con la respuesta a los esteroides o con la supervivencia del injerto. El resultado de la biopsia en rechazo tiene importancia clínica constituyéndose en una guía para el tratamiento de los episodios de rechazo agudo, es así como para 
los pacientes asintomáticos (biopsias por protocolo) o con síntomas leves y puntaje bajo en la biopsia o rechazo leve. Se puede evitar el uso de esteroides a dosis altas e inclusive ser mínima la modificación del tratamiento, usualmente es buena la respuesta al tratamiento con aumento de la inmunosupresión basal o la terapia con pulsos corticosteroides; no ocurre lo mismo cuando un mayor número de episodios de rechazo agudo moderado o severo serán corticorresistentes y en ocasiones requieren tratamiento inmediato con dosis mayores o pulsos de esteroides o con anticuerpos monoclonales, siendo útil para prevenir la progresión a un rechazo crónico ductopénico (32).

Los pacientes con cambios centrilobulares que no cumplen criterios de Banff para rechazo responden muy bien al tratamiento inmunosupresor, semejante al usado en el rechazo usual. El resultado a largo plazo de los pacientes con lesión centrolobulillar aislada es similar a la de los pacientes con cambios centrilobulillares, asociados con el rechazo que presenta la triada clásica. Se ha reportado este hallazgo como un presagio de futuros episodios de rechazo agudo y de rechazo crónico ductopénico $(27,28)$. También se ha descrito fibrosis perivenular, síndrome de Budd-Chiari o enfermedad venooclusiva en casos de lesión perivenular severa (33). El principal problema que se plantea en el diagnóstico diferencial del rechazo agudo celular, es lograr diferenciar entre un paciente con hepatitis B o hepatitis C recurrente ya que pueden compartir criterios o sobreponerse ambas patologías. En el rechazo el tipo de infiltrado es de características heterogéneas, de localización periductal y sin lesión de la placa limitante, además de estos criterios morfológicos pueden ser útiles parámetros clínicos y serológicos que apoyen o permitan descartar la infección viral. En el próximo artículo profundizaremos este tópico.

\section{Claves para el diagnóstico de rechazo agudo celular}

- Inflamación portal: infiltrado heterogéneo con linfocitos activados y eosinófilos

- Lesión ductal: ductulitis de linfocitos y cambios degenerativos ductales

- Endotelialitis o endotelitis: inflamación subendotelial portal y/o central

\section{CONCLUSIÓN}

El patólogo juega un papel importante en la definición de las complicaciones que pueden presentarse en el postrasplante hepático, sobre todo porque el cuadro clínico y los patrones de las anomalías de las enzimas hepáticas, u otros parámetros clínicos o imagenológicos, no son claros $\mathrm{y}$ no pocas veces requieren intervenciones diametralmente opuestas, que llevan a la necesidad de realizar una biopsia hepática, en búsqueda de resolver una duda diagnóstica y obtener un diagnóstico más acertado. Sin embargo, en la interpretación de los hallazgos histopatológicos es fundamental la correlación con la historia clínica completa.

\section{REFERENCIAS}

1. Yu YY, Ji J, Zhou GW, Shen BY, Chen H, Yan JQ et al. Liver biopsy in evaluation of complications following liver transplantation. World journal of gastroenterology. 2004;10(11):1678-81.

2. Coffin CS, Burak KW, Hart J, Gao ZH. The impact of pathologist experience on liver transplant biopsy interpretation. Modern pathology: an official journal of the United States and Canadian Academy of Pathology, Inc. 2006;19(6):832-8.

3. Adeyi O, Fischer SE, Guindi M. Liver allograft pathology: approach to interpretation of needle biopsies with clinicopathological correlation. Journal of clinical pathology. 2010;63(1):47-74.

4. Lucey MR, Terrault N, Ojo L, Hay JE, Neuberger J, Blumberg E, et al. Long-term management of the successful adult liver transplant: 2012 practice guideline by the American Association for the Study of Liver Diseases and the American Society of Transplantation. Liver transplantation: official publication of the American Association for the Study of Liver Diseases and the International Liver Transplantation Society. 2013;19(1):3-26.

5. Stockmann M, Lock JF, Malinowski M, Seehofer D, Puhl G, Pratschke J, et al. How to define initial poor graft function after liver transplantation? - a new functional definition by the LiMAx test. Transplant international: official journal of the European Society for Organ Transplantation. 2010;23(10):1023-32.

6. Uemura $\mathrm{T}$, Randall HB, Sanchez EQ Ikegami $T$, Narasimhan G, McKenna GJ, et al. Liver retransplantation for primary nonfunction: analysis of a 20-year single-center experience. Liver transplantation: official publication of the American Association for the Study of Liver Diseases and the International Liver Transplantation Society. 2007; 13(2):227-33.

7. Lee DD, Croome KP, Shalev JA, Musto KR, Sharma M, Keaveny AP, et al. Early allograft dysfunction after liver transplantation: an intermediate outcome measure for targeted improvements. Annals of hepatology. 2016;15(1):53-60.

8. Lee DD, Singh A, Burns JM, Perry DK, Nguyen JH, Taner CB. Early allograft dysfunction in liver transplantation with donation after cardiac death donors results in inferior survival. Liver transplantation: official publication of the American Association for the Study of Liver Diseases and the International Liver Transplantation Society. 2014;20(12):1447-53.

9. Quesnelle KM, Bystrom PV, Toledo-Pereyra LH. Molecular responses to ischemia and reperfusion in the liver. Archives of toxicology. 2015;89(5):651-7.

10. Abu-Amara M, Yang SY, Tapuria N, Fuller B, Davidson B, Seifalian A. Liver ischemia/reperfusion injury: processes in inflammatory networks: a review. Liver transplantation: offi- 
cial publication of the American Association for the Study of Liver Diseases and the International Liver Transplantation Society. 2010;16(9):1016-32.

11. Jaeschke H, Lemasters JJ. Apoptosis versus oncotic necrosis in hepatic ischemia/reperfusion injury. Gastroenterology. 2003;125(4):1246-57.

12. Bruinsma BG, Wu W, Ozer S, Farmer A, Markmann JF, Yeh $\mathrm{H}$, et al. Warm ischemic injury is reflected in the release of injury markers during cold preservation of the human liver. PloS one. 2015;10(3):e0123421.

13. Hubscher SG. Transplantation pathology. Seminars in diagnostic pathology. 2006;23(3-4):170-81.

14. Lefkowitch JH. Scheuer's Liver biopsy interpretation. Ninth Edition. Elsevier, 2016.

15. Terminology for hepatic allograft rejection. International Working Party. Hepatology (Baltimore, $\mathrm{Md}$ ). 1995;22(2):648-54.

16. Banff schema for grading liver allograft rejection: an international consensus document. Hepatology (Baltimore, Md). 1997;25(3):658-63.

17. Neil DA, Hubscher SG. Current views on rejection pathology in liver transplantation. Transplant international: official journal of the European Society for Organ Transplantation. 2010;23(10):971-83.

18. Demetris AJ, Murase N, Nakamura K, Iwaki Y, Yagihashi A, Valdivia L, et al. Immunopathology of antibodies as effectors of orthotopic liver allograft rejection. Seminars in liver disease. 1992;12(1):51-9.

19. Hubscher SG. Antibody-mediated rejection in the liver allograft. Current opinion in organ transplantation. 2012;17(3):280-6.

20. Della-Guardia B, Almeida MD, Meira-Filho SP, Torres MA, Venco F, Afonso RC, et al. Antibody-mediated rejection: hyperacute rejection reality in liver transplantation? A case report. Transplantation proceedings. 2008;40(3):870-1.

21. Haga H, Egawa H, Shirase T, Miyagawa A, Sakurai T, Minamiguchi $S$, et al. Periportal edema and necrosis as diagnostic histological features of early humoral rejection in ABO-incompatible liver transplantation. Liver transplantation: official publication of the American Association for the Study of Liver Diseases and the International Liver Transplantation Society. 2004;10(1):16-27.

22. Taner T, Stegall MD, Heimbach JK. Antibody-mediated rejection in liver transplantation: current controversies and future directions. Liver transplantation: official publication of the American Association for the Study of Liver Diseases and the International Liver Transplantation Society. 2014;20(5):514-27.

23. O’Leary JG, Michelle Shiller S, Bellamy C, Nalesnik MA, Kaneku H, Jennings LW, et al. Acute liver allograft antibodymediated rejection: an inter-institutional study of significant histopathological features. Liver transplantation: official publication of the American Association for the Study of
Liver Diseases and the International Liver Transplantation Society. 2014;20(10):1244-55.

24. Kozlowski T, Andreoni K, Schmitz J, Hayashi PH, Nickeleit V. Sinusoidal C4d deposits in liver allografts indicate an antibody-mediated response: diagnostic considerations in the evaluation of liver allografts. Liver transplantation: official publication of the American Association for the Study of Liver Diseases and the International Liver Transplantation Society. 2012;18(6):641-58.

25. Song AT, Avelino-Silva VI, Pecora RA, Pugliese V, D’Albuquerque LA, Abdala E. Liver transplantation: fifty years of experience. World journal of gastroenterology. 2014;20(18):5363-74.

26. Torbenson, MS. Biopsy Interpretation of the Liver. Series Editor Jonathan I. Epstein Wolteers Kluwer. Chapter 14th. Transplant Pathology, 2015.

27. Khettry U, Backer A, Ayata G, Lewis WD, Jenkins RL, Gordon FD. Centrilobular histopathologic changes in liver transplant biopsies. Human pathology. 2002;33(3):270-6.

28. Sundaram SS, Melin-Aldana H, Neighbors K, Alonso EM. Histologic characteristics of late cellular rejection, significance of centrilobular injury, and long-term outcome in pediatric liver transplant recipients. Liver transplantation: official publication of the American Association for the Study of Liver Diseases and the International Liver Transplantation Society. 2006;12(1):58-64.

29. Siddiqui I, Selzner N, Hafezi-Bakhtiari S, Marquez MA, Adeyi OA. Infiltrative (sinusoidal) and hepatitic patterns of injury in acute cellular rejection in liver allograft with clinical implications. Modern pathology: an official journal of the United States and Canadian Academy of Pathology, Inc. 2015;28(9):1275-81.

30. Syn WK, Nightingale P, Gunson B, Hubscher SG, Neuberger JM. Natural history of unexplained chronic hepatitis after liver transplantation. Liver transplantation: official publication of the American Association for the Study of Liver Diseases and the International Liver Transplantation Society. 2007; 13(7):984-9.

31. Alexander J, Chu W, Swanson PE, Yeh MM. The significance of plasma cell infiltrate in acute cellular rejection of liver allografts. Human pathology. 2012;43(10):1645-50.

32. Horoldt BS, Burattin M, Gunson BK, Bramhall SR, Nightingale P, Hubscher SG, et al. Does the Banff rejection activity index predict outcome in patients with early acute cellular rejection following liver transplantation? Liver transplantation: official publication of the American Association for the Study of Liver Diseases and the International Liver Transplantation Society. 2006;12(7):1144-51.

33. Sebagh M, Debette M, Samuel D, Emile JF, Falissard B, Cailliez V, et al. «Silent» presentation of veno-occlusive disease after liver transplantation as part of the process of cellular rejection with endothelial predilection. Hepatology (Baltimore, Md). 1999;30(5):1144-50. 